\title{
Solving the Traveling Salesman Problem with Interdiction and Fortification
}

\author{
Leonardo Lozano ${ }^{\mathrm{a}}$, J. Cole Smith ${ }^{\mathrm{a}, *}$, Mary E. Kurz ${ }^{\mathrm{a}}$ \\ ${ }^{a}$ Department of Industrial Engineering, Clemson University, 100 Freeman Hall, PO Box 340920, Clemson, \\ SC 29634-0920.
}

\begin{abstract}
We solve a defender-attacker-defender problem over a traveling salesman problem (TSP), in which the defender first acts to defend a subset of arcs, the attacker then interdicts a subset of undefended arcs (thus increasing their costs), and the defender solves a TSP over the remaining network. Our approach employs an exact approach augmented with a TSP restriction phase to accelerate the convergence of the algorithm. Our computational results show success for the first time in optimally solving defender-attacker-defender TSP problems.
\end{abstract} Keywords: interdiction, integer programming, traveling salesman problem

\section{Introduction}

The traveling salesman problem (TSP) is a well-known $\mathcal{N} \mathcal{P}$-hard problem that seeks a minimum-cost Hamiltonian cycle (tour) over a graph $[1,2]$. We present the traveling salesman problem with interdiction and fortification (TSPIF), modeled as a defender-attacker-defender problem. In the first stage (fortification), the defender fortifies a subset of arcs. In the second stage (attack), an attacker interdicts a subset of unprotected arcs, thus increasing their cost. In the third stage (recourse), the defender solves a TSP defined using the costs resulting from the attack stage. In this context, an attack is not necessarily due to a malicious adversary, but could represent some bounded worst-case scenario on arc cost uncertainty. The TSPIF also arises as an alternative conservative approach to modeling routing problems under uncertainty, in which the road travel times may not be known in advance due to congestion effects [3].

\footnotetext{
*Corresponding author. Department of Industrial Engineering, Clemson University, 100 Freeman Hall, PO Box 340920, Clemson, SC 29634-0920; e-mail: jcsmith@clemson.edu; URL: http://jcsmith.people.clemson.edu
} 
The TSPIF may occur in a defense scenario in which troops need to monitor a set of locations and return to a base. If the troops wish to perform these tasks as quickly as possible, they solve a TSP. An adversary might attempt to impair the troops' movement by degrading (interdicting) roadways or bridges. The adversary's actions could be anticipated by the troops, who secure pathways ahead of time by stationing personnel or other resources to deter interdictions. Hence, the troops act first to fortify arcs, after which the adversary interdicts unfortified arcs, and the troops respond by solving a TSP on the resulting network.

We formally define the TSPIF on a directed graph $\mathcal{G}=(\mathcal{N}, \mathcal{A})$, where $\mathcal{N}$ is the set of nodes and $\mathcal{A} \subset \mathcal{N} \times \mathcal{N}$ is the set of arcs. For each arc $(i, j) \in \mathcal{A}$, let $c_{i j} \geq 0$ be the cost of traversing an uninterdicted arc and $d_{i j} \geq 0$ be the additional cost (delay) incurred when traversing an interdicted arc. Thus, the total cost of traversing an interdicted arc is $c_{i j}+d_{i j}$. Let $\mathbf{w} \in \mathcal{W}$ be the fortification decision variables, where $\mathcal{W} \equiv\{\mathbf{w}|\mathbf{T w} \leq \mathbf{b}, \mathbf{w} \in\{0,1\}| \mathcal{A} \mid\}$ ensures that the variables are binary and enforces a set of linear constraints that limits the extent to which the defender can fortify arcs. Let $\mathbf{x} \in \mathcal{X}(\mathbf{w})$ be the attack decision variables, where $\mathcal{X}(\mathbf{w}) \equiv\left\{\mathbf{x} \mid \mathbf{T}^{\prime} \mathbf{x} \leq \mathbf{b}^{\prime}, x_{i j} \leq 1-w_{i j}, \forall(i, j) \in \mathcal{A}, \mathbf{x} \in\{0,1\}^{|\mathcal{A}|}\right\}$ forces the $\mathbf{x}$-variables to be binary, ensures that only unfortified arcs are interdicted, and imposes a set of linear constraints that models the ability of the attacker to interdict arcs. Finally, let $\mathbf{y}$ be a vector of binary arc-selection variables such that $y_{i j}=1$ if $\operatorname{arc}(i, j)$ is used in the optimal tour identified for the recourse problem, and $y_{i j}=0$ otherwise, for all $(i, j) \in \mathcal{A}$. We restrict $\mathbf{y} \in \mathcal{Y}$, where $\mathcal{Y}$ includes the set of binary vectors $\mathbf{y}$ that correspond to TSP solutions in $\mathcal{G}$. The TSPIF can be formally stated as:

$$
z^{*}=\min _{\mathbf{w} \in \mathcal{W}} \max _{\mathbf{x} \in \mathcal{X}(\mathbf{w})} \min _{\mathbf{y} \in \mathcal{Y}} \sum_{(i, j) \in \mathcal{A}}\left(c_{i j}+d_{i j} x_{i j}\right) y_{i j}
$$

where in the objective function (1), the original cost of any arc is increased by $d_{i j}$ when the arc is attacked (i.e., $x_{i j}=1$ ).

Previous studies on defender-attacker-defender problems include enumerative schemes $[4,5,6,7]$, decomposition approaches based on supervalid inequalities $[8,9]$, and approaches based on strong duality $[10,11,12]$. The latter approaches combine the second-and thirdstage problems by taking the dual of the third-stage problem, and then solve the resulting problem using a Benders' decomposition algorithm in which the subproblem is a mixed-integer 
programming problem (MIP). Since the TSP is a nonconvex combinatorial problem, we do not combine the second- and third-stage problems, because no polynomial-size strong dual formulation for the TSP is known to exist. In response to this problem, Lozano and Smith [13] propose a backward sampling framework (BSF) for interdiction problems with fortification in which the recourse problem can take any form. They solve defender-attacker-defender games played over shortest path (SPIF) and capacitated lot sizing problems (CLSIF). The BSF significantly outperforms prior approaches for solving the SPIF, and yields an effective mechanism for solving interdiction and fortification problems. However, the design of practically effective algorithms for interdiction and fortification problems defined over a strongly $\mathcal{N} \mathcal{P}$-hard problem like the TSP is still an open research question.

In this paper we explore the solution of the TSPIF via the BSF. Our contribution analyzes two problem restrictions, where both restrictions serve as a heuristic for the TSPIF and model the situation in which the defender lacks the computational resources to compute an optimal response to an attack. We also demonstrate that these restrictions are instrumental in reducing computational time for solving the TSPIF within an exact two-phase approach. We then use these developments to tailor a BSF-based approach for this problem, and demonstrate the efficacy of our approach using TSP instances from the literature.

\section{The Backward Sampling Framework for the TSPIF}

Our algorithm is composed of an outer cutting-plane approach that optimizes the defense decisions and an inner sampling-based optimization algorithm that solves the corresponding two-level problem. The inner algorithm restricts the defender to select a recourse decision from a sample of TSP tours over $\mathcal{G}$ and iteratively refines the sample to force finite convergence to an optimal solution.

\subsection{Solving the TSPIF}

Our outer algorithm evaluates defense vectors $\mathbf{w} \in \mathcal{W}$ that eliminate critical attacks. An attack $\hat{\mathbf{x}}$ is critical if

$$
z^{R}(\hat{\mathbf{x}})=\min _{\mathbf{y} \in \mathcal{Y}} \sum_{(i, j) \in \mathcal{A}}\left(c_{i j}+d_{i j} \hat{x}_{i j}\right) y_{i j} \geq z^{*}
$$


i.e., if the objective function value of an optimal TSP tour given an attack vector $\hat{\mathbf{x}}$ is greater than or equal to $z^{*}$. We add a covering constraint (also known as a "no-good constraint" [14]) of the form $\mathbf{w}^{\top} \hat{\mathbf{x}} \geq 1$ to the fortification problem for each critical attack $\hat{\mathbf{x}}$, forcing the defender to protect at least one of the arcs interdicted by $\hat{\mathbf{x}}$.

Our inner algorithm solves the corresponding two-level interdiction problem for every defense vector $\hat{\mathbf{w}} \in \mathcal{W}$ selected by the outer algorithm:

$$
\mathcal{Q}(\hat{\mathbf{w}}): z^{I}(\hat{\mathbf{w}})=\max _{\mathbf{x} \in \mathcal{X}(\hat{\mathbf{w}})} \min _{\mathbf{y} \in \mathcal{Y}} \sum_{(i, j) \in \mathcal{A}}\left(c_{i j}+d_{i j} x_{i j}\right) y_{i j}
$$

We assume that $\mathcal{X}(\mathbf{w}) \neq \emptyset, \forall \mathbf{w} \in \mathcal{W}$, and so an optimal solution to (3) must exist.

Since problem $\mathcal{Q}(\hat{\mathbf{w}})$ does not belong to $\mathcal{N} \mathcal{P}$ unless $\mathcal{P}=\mathcal{N} \mathcal{P}$, our algorithm relies on a sampling procedure that iteratively solves a series of restricted problems whose solutions converge to an optimal solution to $\mathcal{Q}(\hat{\mathbf{w}})$. Let $\hat{\mathcal{Y}} \subseteq \mathcal{Y}$ be a sample of TSP tours over $\mathcal{G}$ and

$$
\mathcal{Q}(\hat{\mathbf{w}}, \hat{\mathcal{Y}}): z^{I}(\hat{\mathbf{w}}, \hat{\mathcal{Y}})=\max _{\mathbf{x} \in \mathcal{X}(\hat{\mathbf{w}})} \min _{\mathbf{y} \in \hat{\mathcal{Y}}} \sum_{(i, j) \in \mathcal{A}}\left(c_{i j}+d_{i j} x_{i j}\right) y_{i j}
$$

be the restricted problem in which recourse decisions are restricted to $\hat{\mathcal{Y}}$. For a given $\hat{\mathbf{w}} \in \mathcal{W}$ and a sample of tours $\hat{\mathcal{Y}} \subseteq \mathcal{Y}$, we formulate $\mathcal{Q}(\hat{\mathbf{w}}, \hat{\mathcal{Y}})$ as a MIP. Let $\mathcal{T}^{k}$ be the set of arcs corresponding to the $k^{\text {th }}$ tour in sample $\hat{\mathcal{Y}}$, and let $c\left(\mathcal{T}^{k}\right)$ denote its cost. We formulate $\mathcal{Q}(\hat{\mathbf{w}}, \hat{\mathcal{Y}})$ as follows:

$$
\begin{aligned}
z^{I}(\hat{\mathbf{w}}, \hat{\mathcal{Y}})=\max & z \\
\text { s.t. } & z \leq c\left(\mathcal{T}^{k}\right)+\sum_{(i, j) \in \mathcal{T}^{k}} d_{i j} x_{i j} \quad \forall \mathcal{T}^{k} \in \hat{\mathcal{Y}} \\
& \mathbf{x} \in \mathcal{X}(\hat{\mathbf{w}}) .
\end{aligned}
$$

The objective function (5) maximizes $z$, which is constrained by (6) to be no more than the least-cost tour in $\hat{\mathcal{Y}}$, after considering additional costs caused by arc interdiction. Constraints (7) restrict $\mathbf{x}$ to the set of feasible attacks.

For any $\hat{\mathbf{w}} \in \mathcal{W}$ and sample of tours $\hat{\mathcal{Y}} \subseteq \mathcal{Y}, z^{I}(\hat{\mathbf{w}}, \hat{\mathcal{Y}})$ is an upper bound on $z^{I}(\hat{\mathbf{w}})$, which is in turn an upper bound on $z^{*}$. Lower bounds can be obtained by solving a TSP to calculate 
$z^{R}(\hat{\mathbf{x}})$ for any $\hat{\mathbf{x}} \in \mathcal{X}(\hat{\mathbf{w}})$. Combining these inequalities, we obtain:

$$
z^{R}(\hat{\mathbf{x}}) \leq z^{I}(\hat{\mathbf{w}}) \leq z^{I}(\hat{\mathbf{w}}, \hat{\mathcal{Y}}), \forall \hat{\mathbf{w}} \in \mathcal{W}, \hat{\mathbf{x}} \in \mathcal{X}(\hat{\mathbf{w}}), \hat{\mathcal{Y}} \subseteq \mathcal{Y}
$$

Thus, if $(\hat{\mathbf{x}}, \hat{\mathbf{y}})$ solves $\mathcal{Q}(\hat{\mathbf{w}}, \hat{\mathcal{Y}})$ and $z^{I}(\hat{\mathbf{w}}, \hat{\mathcal{Y}})=z^{R}(\hat{\mathbf{x}})$, then $(\hat{\mathbf{x}}, \hat{\mathbf{y}})$ solves $\mathcal{Q}(\hat{\mathbf{w}})[13]$.

Algorithm 1 presents the proposed approach. Let $\mathcal{C}$ be the set of covering constraints added to the (outer) fortification problem and $\mathcal{W}(\mathcal{C})=\{\mathbf{w} \in \mathcal{W} \mid \mathbf{w}$ satisfies all constraints in $\mathcal{C}$ \}. The algorithm starts with an empty set of covering constraints and a global upper bound $\bar{z}=\infty$. Line 2 selects an initial sample of tours over $\mathcal{G}$. The outer while-loop (line 4 ) is executed until the fortification problem becomes infeasible. Line 5 selects a feasible defense $\hat{\mathbf{w}}$ and lines $6-21$ solve the corresponding problem $\mathcal{Q}(\hat{\mathbf{w}})$ with our proposed sampling approach. The inner while-loop (line 7) is executed until the global upper bound cannot be further reduced by the current choice of $\hat{\mathbf{w}}$. Line 9 obtains an upper bound on $z^{I}(\hat{\mathbf{w}})$ by solving the restricted problem $\mathcal{Q}\left(\hat{\mathbf{w}}, \hat{\mathcal{Y}}^{i}\right)$ and obtaining an attack vector $\hat{\mathbf{x}} \in \mathcal{X}(\hat{\mathbf{w}})$. Line 10 solves a TSP given the fixed attack $\hat{\mathbf{x}}$. The optimal tour, $\hat{\mathbf{y}}^{*}$, identified in this step yields a lower bound. Line 11 defines the sample at the next iteration as the solutions in the previous sample along with $\hat{\mathbf{y}}^{*}$. Line 12 checks if $U B_{i}$ reduces the current global upper bound; if so, then line 13 updates the global upper bound, and line 14 removes from the sample all tours whose cost is greater than $\bar{z}$. Line 15 determines if attack $\hat{\mathbf{x}}$ is critical by checking if $L B_{i} \geq \bar{z}$, and if so, line 16 adds a covering constraint to the fortification problem. Finally, if the optimality condition is satisfied (line 18), then line 19 updates the incumbent solution. 


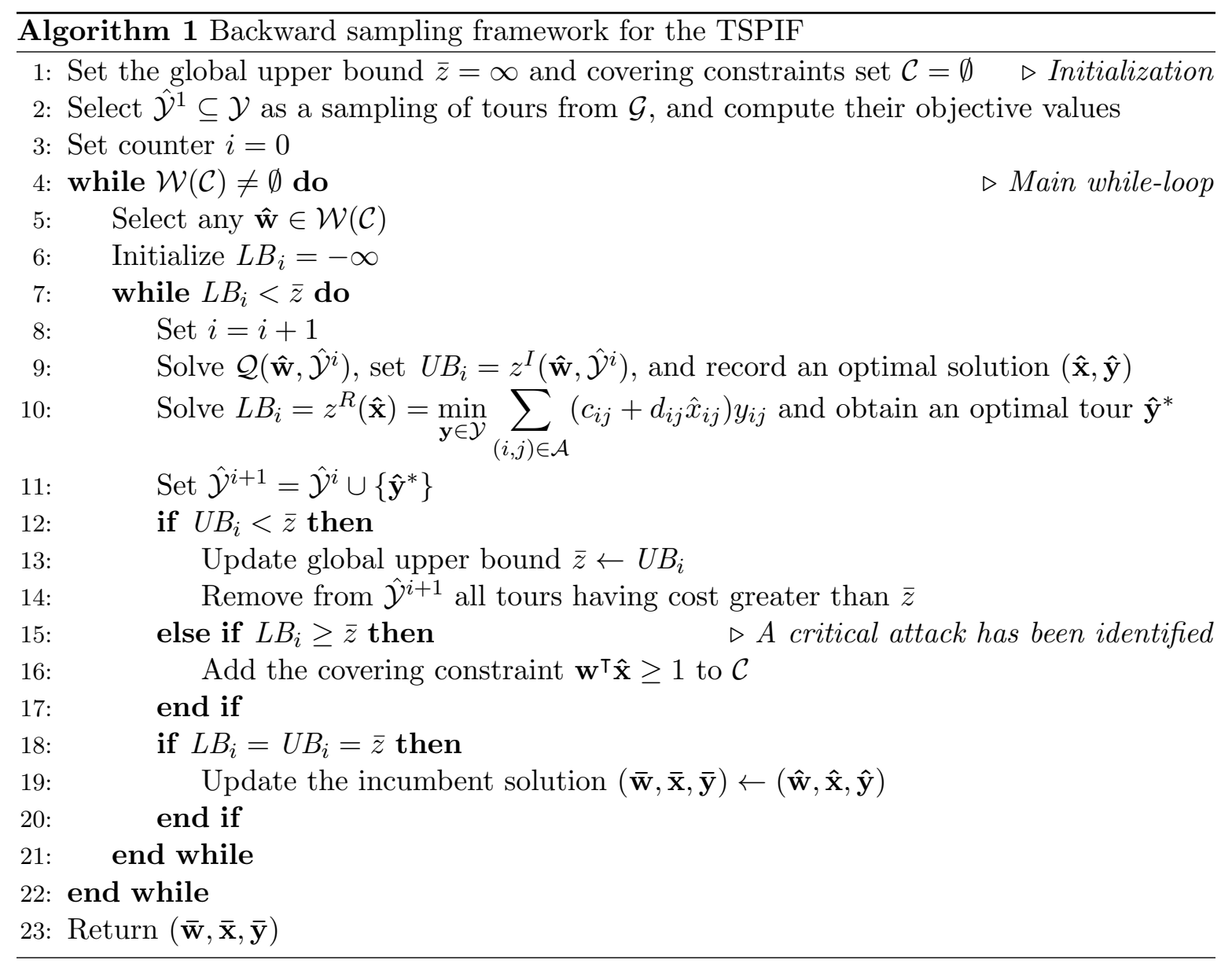

\subsection{Sampling TSP Tours}

The only condition on the initial sample to ensure that our algorithm terminates with an optimal solution is that $\hat{\mathcal{Y}}^{1} \subseteq \mathcal{Y}$. However, $\hat{\mathcal{Y}}^{1}$ impacts the performance of the BSF since both the tightness of the upper bounds obtained by solving restricted problems $\mathcal{Q}\left(\hat{\mathbf{w}}, \hat{\mathcal{Y}}^{i}\right)$ and the number of constraints in formulation (5)-(7) depend on the choice of $\hat{\mathcal{Y}}^{1}$.

We now describe desirable features for a choice of $\hat{\mathcal{Y}}^{1}$. Tours in $\hat{\mathcal{Y}}^{1}$ should be diverse in the sense that they do not contain too many of the same arcs, or else the attacker could interdict many tours in the sample by interdicting a few arcs common to those tours. Tours in $\hat{\mathcal{Y}}^{1}$ should also be optimal or near-optimal solutions to the TSP when $\hat{\mathbf{x}}=\mathbf{0}$. Finally, if $\left|\hat{\mathcal{Y}}^{1}\right|$ is too large, then formulation (5)-(7) will be large as well, and may potentially be too difficult to solve. 
Attempting to achieve a balance between the desirable features listed, we propose a genetic algorithm (GA) based on the NSGA-II framework [15] in which each solution has two objectives, both of which are to be minimized. The first objective is the tour length with respect to the uninterdicted graph. The second objective is a measure of the individual solution's similarity to some reference set of TSP tours. We include in our reference set all tours whose length is not more than $\epsilon$ percent greater than the best tour-length seen so far. We compute our second objective as the number of times each solution arc appears in the reference set, divided by the total number of arcs in the set population. A solution that has no arcs in common with any tour in the reference set is in some manner "maximally different" and is desirable, having a second objective of 0 . (The implementation details for the GA are available from the authors.)

Alternatively, one simple option is to seed $\hat{\mathcal{Y}}^{1}$ with one TSP tour. In this case we solve the TSP when $\hat{\mathbf{x}}=\mathbf{0}$, and use only that tour in our initial sample.

\subsection{Alternative Restrictions for the Recourse Problem}

We now present two restrictions for the recourse problem that model the case in which the defender must compute a quick response to an attack, rather than expending the computational resources required to compute an optimal response. These restrictions are also instrumental in devising a more computationally effective exact TSPIF algorithm.

The first restriction is inspired by very large-scale neighborhood search algorithms [16]. We start with a base tour $\mathbf{y}^{*}$ obtained by solving the TSP to optimality given $\operatorname{costs} c_{i j}, \forall(i, j) \in$ $\mathcal{A}$. For the symmetric case, the defender recourse responses are restricted to belong to the set of tours that can be obtained by performing a series of so-called disjoint 2-opt swaps on $\mathbf{y}^{*}$. (See Remark 1 for an extension of this idea for the asymmetric case.) To understand the concept of disjoint 2-opt swaps, we first order the nodes in tour $\mathbf{y}^{*}$ as $v_{(1)}, v_{(2)}, \ldots, v_{(|\mathcal{N}|)}$. Let $v_{(|\mathcal{N}|+1)} \equiv v_{(1)}$. A 2-opt swap is performed by identifying two tour indices $i$ and $j$, where $i \geq 1, j \leq|\mathcal{N}|+1$, and $i+3 \leq j$. The tour formed by a 2 -arc swap replaces arcs $\left(v_{(i)}, v_{(i+1)}\right)$ and $\left(v_{(j-1)}, v_{(j)}\right)$ with $\operatorname{arcs}\left(v_{(i)}, v_{(j-1)}\right)$ and $\left(v_{(i+1)}, v_{(j)}\right)$ in the original tour. Arcs $\left(v_{(k)}, v_{(k+1)}\right), k=i+1, \ldots, j-2$, would now be traversed in the opposite direction after the 
symmetric 2-opt arc swap. (See Figure 1 for an illustration.) A set of 2-opt swaps are disjoint if the 2-opt swaps are performed over indices $\left(i_{1}, j_{1}\right),\left(i_{2}, j_{2}\right), \ldots,\left(i_{k}, j_{k}\right)$ such that $j_{h} \leq i_{h+1}, \forall h=1, \ldots, k-1$.

We model the disjoint 2-opt swap restriction on $\mathbf{y}^{*}$ by transforming the recourse problem into a shortest path problem defined over a new graph $\mathcal{G}^{\prime}=\left(\mathcal{N}^{\prime}, \mathcal{A}^{\prime}\right)$. The set of nodes $\mathcal{N}^{\prime}=\{1, \ldots,|\mathcal{N}|+1\}$ represents each ordered node in tour $\mathbf{y}^{*}$, where $|\mathcal{N}|+1$ is a duplicate of the first node. The set of $\operatorname{arcs} \mathcal{A}^{\prime}=\mathcal{A}_{1}^{\prime} \cup \mathcal{A}_{2}^{\prime}$ comprises two kinds of arcs. Arcs in $\mathcal{A}_{1}^{\prime}=\left\{(i, i+1)\left|i \in \mathcal{N}^{\prime}, i \leq\right| \mathcal{N} \mid\right\}$ correspond to arcs in the original tour $\mathbf{y}^{*}$. Accordingly, we define their cost as $c_{i j}^{\prime}=c_{v_{(i)} v_{(j)}}$ and delay for a given attack $\mathbf{x}$ as $d_{i j}^{\prime}=d_{v_{(i)} v_{(j)}} x_{v_{(i)} v_{(j)}}$, for all $(i, j) \in \mathcal{A}_{1}^{\prime}$. Arcs in $\mathcal{A}_{2}^{\prime}=\{(i, j)|\forall i=1, \ldots,| \mathcal{N}|-2, j=i+3, \ldots,| \mathcal{N} \mid+1\}$ represent a 2-opt swap as illustrated in Figure 1. For arc $(i, j) \in \mathcal{A}_{2}^{\prime}$ the cost and delay for a given attack $\mathbf{x}$ are defined as:

$$
\begin{array}{ll}
c_{i j}^{\prime}=c_{v_{(i)} v_{(j-1)}}+c_{v_{(i+1)} v_{(j)}}+\sum_{k=i+2}^{j-1} c_{v_{(k)} v_{(k-1)}} & \forall(i, j) \in \mathcal{A}_{2}^{\prime} \\
d_{i j}^{\prime}=d_{v_{(i)} v_{(j-1)}} x_{v_{(i)} v_{(j-1)}}+d_{v_{(i+1)} v_{(j)}} x_{v_{(i+1)} v_{(j)}}+\sum_{k=i+2}^{j-1} d_{v_{(k)} v_{(k-1)}} x_{v_{(k)} v_{(k-1)}} & \forall(i, j) \in \mathcal{A}_{2}^{\prime},
\end{array}
$$

respectively. Note that the third term in equations (8) and (9) accounts for the arcs traversed from $v_{(i+1)}$ to $v_{(j-1)}$ in the original tour, though in the reverse direction.

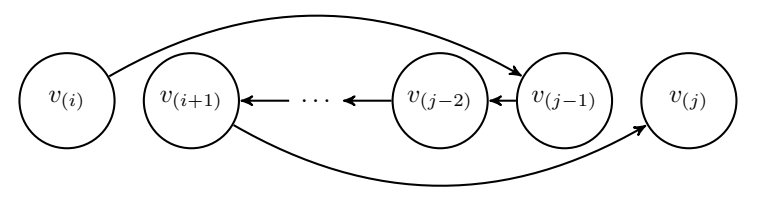

Figure 1: Graphical representation of a 2-arc swap for the symmetric case

Every path from 1 to $|\mathcal{N}|+1$ in $\mathcal{G}^{\prime}$ corresponds to a TSP tour in the original graph $\mathcal{G}$. These paths encode the original tour $\mathbf{y}^{*}$ along with all solutions that can be obtained via disjoint 2-opt swaps from that tour.

Remark 1 . For the asymmetric case, arcs in $\mathcal{A}_{1}^{\prime}$ are given as before. $\operatorname{Arcs}(i, j) \in \mathcal{A}_{2}^{\prime}$ rep- 
resent a 3 -arc swap that replaces arcs $\left(v_{(i)}, v_{(i+1)}\right),\left(v_{(q)}, v_{(q+1)}\right)$, and $\left(v_{(j-1)}, v_{(j)}\right)$ with arcs $\left(v_{(i)}, v_{(q+1)}\right),\left(v_{(j-1)}, v_{(i+1)}\right)$, and $\left(_{(q)}, v_{(j)}\right)$ in the original tour, where

$$
q \in \underset{i+1 \leq \bar{q} \leq j-2}{\operatorname{argmin}}\left\{c_{v_{(i)} v_{(\bar{q}+1)}}+c_{v_{(j-1)} v_{(i+1)}}+c_{v_{(\bar{q})} v_{(j)}}-c_{v_{(i)} v_{(i+1)}}-c_{v_{(q)}, v_{(q+1)}}-c_{v_{(j-1)} v_{(j)}}\right\} .
$$

Note that $q$ is chosen in (10) so that the perturbed route corresponding to arc $(i, j) \in \mathcal{A}_{2}^{\prime}$ is as close to optimal as possible with respect to the uninterdicted graph.

Cost and delay attributes are defined analogous to the symmetric case:

$$
\begin{aligned}
c_{i j}^{\prime} & =c_{v_{(i)} v_{(q+1)}}+c_{v_{(j-1)} v_{(i+1)}}+c_{v_{(q)} v_{(j)}}+\sum_{k=q+1}^{j-2} c_{v_{(k)} v_{(k+1)}}+\sum_{k=i+1}^{q-1} c_{v_{(k)} v_{(k+1)}} & \\
d_{i j}^{\prime} & =d_{v_{(i)} v_{(q+1)}} x_{v_{(i)} v_{(q+1)}}+d_{v_{(j-1)} v_{(i+1)}} x_{v_{(j-1)} v_{(i+1)}}+d_{v_{(q)} v_{(j)}} x_{v_{(q)} v_{(j)}} & \\
& +\sum_{k=q+1}^{j-2} d_{v_{(k)} v_{(k+1)}} x_{v_{(k)} v_{(k+1)}}+\sum_{k=i+1}^{q-1} d_{v_{(k)} v_{(k+1)}} x_{v_{(k)} v_{(k+1)}} & \forall(i, j) \in \mathcal{A}_{2}^{\prime}
\end{aligned}
$$

We also consider a second restriction that constrains the defender to solve the recourse problem using the Lin-Kernighan heuristic (LKH) [17]. Note that the first restriction is modeled as a network flow problem, which can then be solved using existing network interdiction and fortification algorithms $[4,18]$. On the other hand, the second restriction will most likely yield a stronger upper bound given the success of the LKH in obtaining near-optimal TSP solutions, but the restriction cannot practically be modeled as a linear program.

\subsection{Two-Phase Approach}

We devise a two-phase approach that first solves a restriction of the TSPIF to identify a set of covering constraints, an initial sample of tours, and an upper bound on $z^{*}$. This first phase is based on the solution of a heuristic TSP restriction, and provides a warm start to exactly solve the problem using Algorithm 1 in a second phase.

Algorithm 2 describes our proposed two-phase approach. Line 1 solves a restriction of the TSPIF using a variation of Algorithm 1 in which one of the proposed restrictions in Section

2.3 is used to compute recourse solutions. We record an optimal solution, $\left(\mathbf{w}^{0}, \mathbf{x}^{0}, \mathbf{y}^{0}\right)$, and the 
set of all critical attacks explored, $\hat{\mathcal{X}}$, solving this restricted problem. Note that even though attacks in $\hat{\mathcal{X}}$ are critical for the restricted problem, they are not necessarily critical for the original (exact) problem. Line 2 solves to optimality the interdiction problem corresponding to $\mathbf{w}^{0}$ (using our inner sampling-based algorithm) and line 3 updates the upper bound and incumbent solution accordingly. Lines $5-11$ explore all attacks $\hat{\mathbf{x}} \in \hat{\mathcal{X}}$ to generate the initial sample and possibly identify covering constraints. For every $\hat{\mathbf{x}} \in \hat{\mathcal{X}}$, line 6 solves a TSP to find an optimal recourse tour $\hat{\mathbf{y}}^{*}$ and calculates $z^{R}(\hat{\mathbf{x}})$. Line 7 adds $\hat{\mathbf{y}}^{*}$ into the initial sample. Line 8 determines if attack $\hat{\mathbf{x}}$ is critical by checking if $z^{R}(\hat{\mathbf{x}}) \geq \bar{z}$. If so, then line 9 adds a covering constraint to the fortification problem. Finally, line 12 continues solving the TSPIF using Algorithm 1, starting with an initial sample $\hat{\mathcal{Y}}^{1}$, a covering constraint set $\mathcal{C}$, and an upper bound $\bar{z}$.

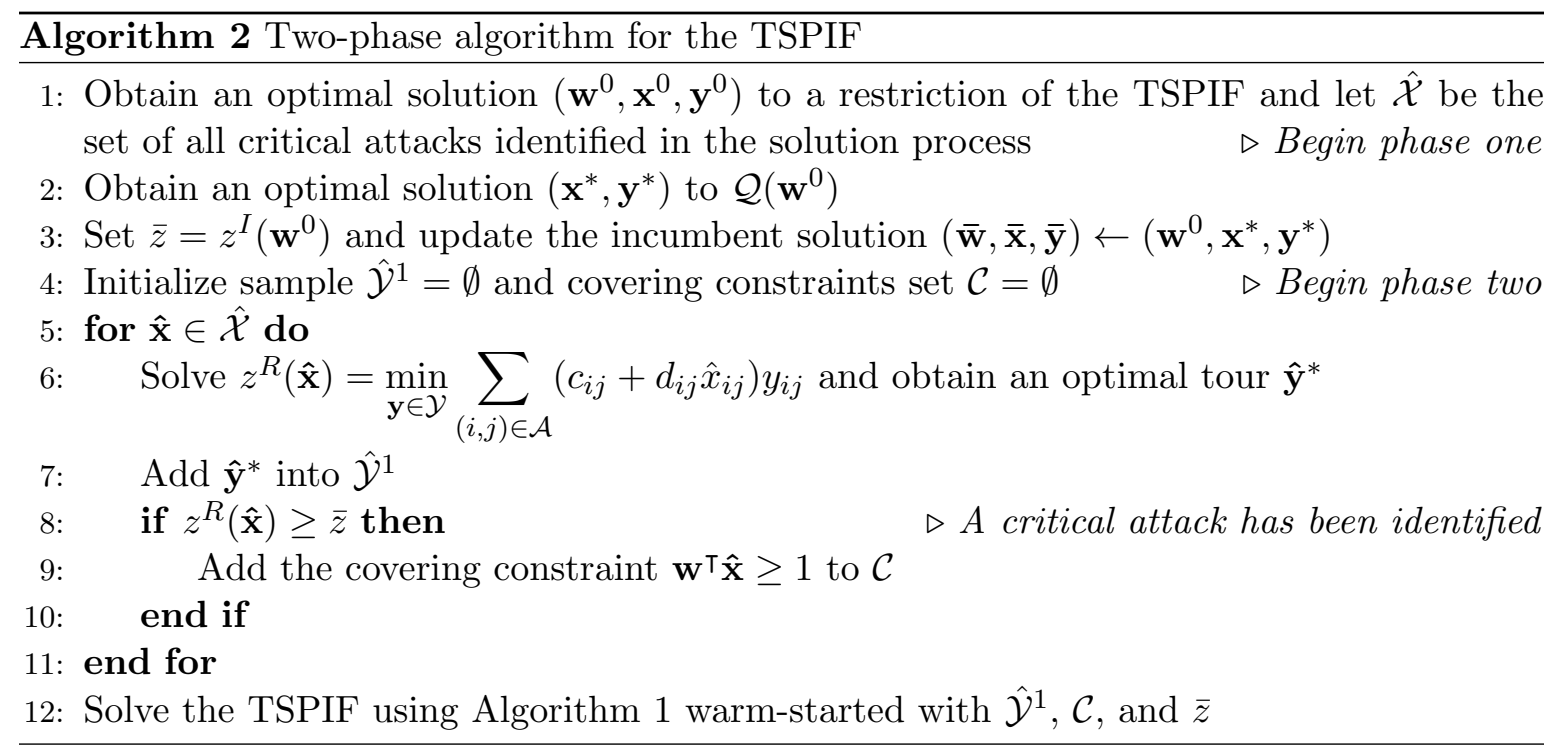

\section{Computational Results}

We coded our algorithm in Java, using Eclipse SDK version 4.4.2, and executed all computational experiments on a machine having an Intel Core i7-3537U CPU (two cores) running at $2.00 \mathrm{GHz}$ with $8 \mathrm{~GB}$ of RAM on Windows 8 . We solve the TSP instances using CONCORDE $[19,20]$, all other optimization problems using Gurobi 5.6.0, and use the LKH implementation 
provided by [21].

Our set of test instances consists of 100 instances derived from 10 networks (5 symmetric and 5 asymmetric) from TSPLIB [22]. In every instance the cost coefficient for $\operatorname{arc}(i, j) \in \mathcal{A}$ corresponds to the distance between nodes $i$ and $j$ in the original network. The delay coefficient for $\operatorname{arc}(i, j) \in \mathcal{A}$ is initially taken to be a random integer uniformly distributed between $\left[1, c_{i j}\right]$. We generate 10 instances with random arc delay coefficients for each of the original networks. We define the defender's feasible re-

gion as $\mathcal{W} \equiv\left\{\mathbf{w} \mid \mathbf{e}^{\top} \mathbf{w} \leq Q, \mathbf{w} \in\{0,1\}^{|\mathcal{A}|}\right\}$, which enforces a cardinality constraint on the number of fortified arcs and ensures that the variables are binary. We also define $\mathcal{X}(\mathbf{w}) \equiv\left\{\mathbf{x} \mid \mathbf{e}^{\top} \mathbf{x} \leq B, x_{i j} \leq 1-w_{i j}, \forall(i, j) \in \mathcal{A}, \mathbf{x} \in\{0,1\}^{|\mathcal{A}|}\right\}$, which ensures that a maximum of $B$ unfortified arcs are interdicted, and forces the $\mathbf{x}$-variables to be binary.

\subsection{Solving the TSPIF}

We compared four versions of the proposed algorithm. The first one (one-tour sampling) initializes the sample as a single tour that optimizes the TSP when no arcs have been interdicted. The second one (GA sampling) implements the proposed GA sampling scheme. The third one (two-phase 2-opt) implements the two-phase algorithm in Section 2.4 with the 2-opt restriction, and the fourth one (two-phase LKH) implements the two-phase algorithm with the LKH restriction. We solve each instance three times with different budget configurations $(Q, B)$ in $\{(3,3),(5,4),(4,5)\}$ for a total of 300 experiments for each version of the algorithm.

Table 1 shows the results for these experiments. The first five rows present results for symmetric instances and the last five rows for asymmetric instances. The columns present the average CPU time in seconds obtained over 10 instances derived from the same network (Avg), the largest CPU time obtained over those runs (Max), and the number of instances solved within a four-hour time limit (\# solved) for the four versions of the algorithm. We calculate the average CPU time only among the instances solved within the time limit and report an overall CPU time average for the symmetric and asymmetric instances. For each row, the best average and maximum CPU times are highlighted in bold. 


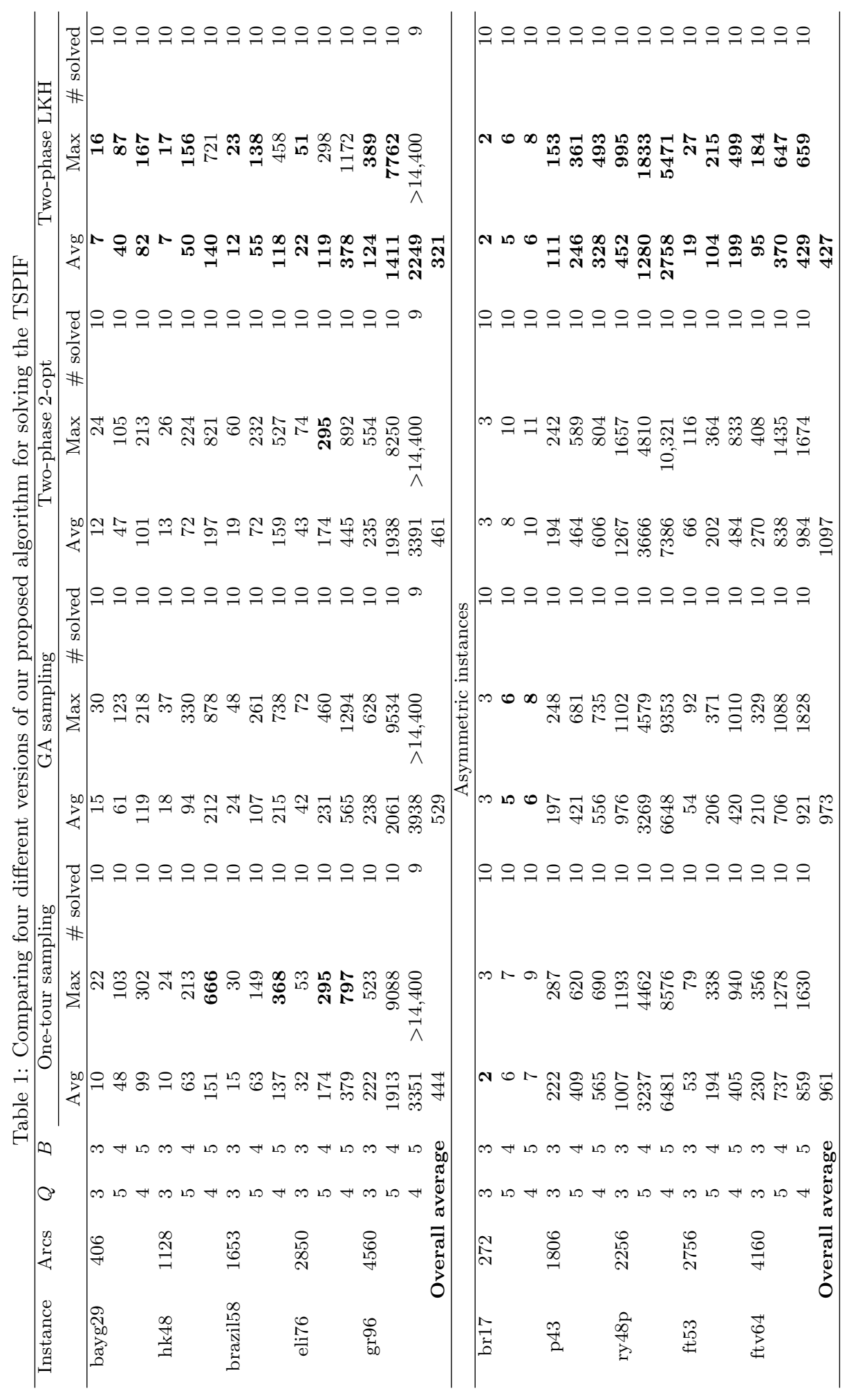


Table 1 shows that two-phase LKH outperforms the other algorithms. For the symmetric instances, two-phase LKH is on average about $40 \%$ faster than one-tour sampling and two-phase 2-opt, and about $65 \%$ faster than GA sampling. However, there is one instance from network gr96 that none of the algorithms solve within the time limit. For the harder asymmetric instances, two-phase LKH is on average more than two times faster than the other algorithms. The maximum CPU times follow a similar behavior.

\subsection{Assessing the Effectiveness of the Proposed Restrictions}

Table 2 compares two-phase 2-opt and two-phase LKH when $Q=4$ and $B=5$, which was the most difficult budget configuration in Table 1. We omitted instance gr96, which was not solved to optimality. Column " $z^{*}$ " presents the average optimal objective value obtained over the instances derived from the same network. The remaining columns show the average upper bound obtained at the end of phase one $(\bar{z})$, the average objective function value gap, calculated as $\left(\bar{z}-z^{*}\right) / z^{*} \times 100$ (\% Gap), the average CPU time in seconds for phase one (I), the average CPU time for phase two (II), the average total CPU time (Total), and the number of covering constraints added at the end of phases one and two (Cuts).

Table 2: Comparing the performance of the proposed restrictions within the two-phase approach

\begin{tabular}{|c|c|c|c|c|c|c|c|c|c|c|c|c|c|}
\hline \multirow{3}{*}{ Instance } & \multirow{3}{*}{$z^{*}$} & \multicolumn{6}{|c|}{ Two-phase 2-opt } & \multicolumn{6}{|c|}{ Two-phase LKH } \\
\hline & & \multirow[t]{2}{*}{$\overline{\bar{z}}$} & \multirow[t]{2}{*}{$\%$ Gap } & \multicolumn{2}{|c|}{ Time $(\mathrm{s})$} & \multicolumn{2}{|c|}{ Cuts } & \multirow[t]{2}{*}{$\bar{z}$} & \multirow[t]{2}{*}{$\%$ Gap } & \multicolumn{2}{|c|}{ Time $(\mathrm{s})$} & \multicolumn{2}{|c|}{ Cuts } \\
\hline & & & & I & II & I & II & & & $\mathrm{I}$ & II & $\mathrm{I}$ & II \\
\hline bayg29 & 1702 & 1722 & 1.20 & 17 & 84 & 9 & 21 & 1702 & 0 & 80 & 2 & 23 & 0 \\
\hline hk48 & 12,057 & 12,140 & 0.68 & 60 & 137 & 5 & 25 & 12,057 & 0 & 136 & 4 & 26 & 0 \\
\hline brazil58 & 26,349 & 26,477 & 0.49 & 49 & 110 & 6 & 22 & 26,349 & 0 & 112 & 6 & 29 & 0 \\
\hline eli76 & 560 & 563 & 0.70 & 44 & 401 & 1 & 36 & 560 & 0 & 365 & 13 & 39 & 0 \\
\hline \multirow[t]{2}{*}{ gr96 } & 56,951 & 57,183 & 0.41 & 749 & 2643 & 4 & 26 & 56,951 & 0 & 2157 & 92 & 28 & 0 \\
\hline & Overall & verage & 0.69 & 184 & 675 & 5 & 26 & & 0 & 570 & 24 & 29 & 0 \\
\hline \multicolumn{14}{|c|}{ Asymmetric instances } \\
\hline br17 & 40 & 41 & 1.75 & 2 & 8 & 1 & 15 & 40 & 0 & 5 & 2 & 16 & 0 \\
\hline $\mathrm{p} 43$ & 5625 & 5630 & 0.08 & 107 & 499 & 1 & 19 & 5626 & 0.01 & 72 & 257 & 19 & 2 \\
\hline ry $48 \mathrm{p}$ & 14,884 & 14,991 & 0.72 & 1188 & 6198 & 1 & 23 & 14,885 & 0.01 & 610 & 2148 & 21 & 2 \\
\hline $\mathrm{ft} 53$ & 7185 & 7243 & 0.81 & 78 & 407 & 3 & 28 & 7185 & 0 & 167 & 32 & 27 & 0 \\
\hline ftv64 & 1921 & 1929 & 0.41 & 140 & 843 & 2 & 34 & 1922 & 0.05 & 47 & 381 & 37 & 1 \\
\hline \multicolumn{3}{|c|}{ Overall average } & 0.75 & 303 & 1591 & 2 & 24 & & 0.01 & 180 & 564 & 24 & 1 \\
\hline
\end{tabular}

Table 2 shows that for symmetric instances, two-phase 2-opt quickly obtains near-optimal heuristic solutions with an objective function gap less than $1 \%$ on average. However, the total time to find an optimal solution by two-phase 2-opt is larger than the time required by two- 
phase LKH, which finds an optimal solution for every instance at the end of phase one. The 2-opt restriction identifies only a small number of covering constraints compared to the LKH restriction.

We conclude that for symmetric instances the 2-opt restriction is the best choice for a stand-alone heuristic, and the LKH restriction is better when embedded in our two-phase exact algorithm. For the asymmetric instances, the LKH restriction outperforms the 2-opt restriction both as a stand-alone heuristic and within our exact algorithm, finding heuristic solutions with a smaller average gap in less computational time. Both algorithms require considerably more time for phase two on the asymmetric instances.

\subsection{Sensitivity Analysis}

We conduct additional sensitivity analysis experiments related to the defender's budget, $Q$, the attacker's budget, $B$, and the range of the arc delay coefficient. For this purpose, we select a subset of 10 symmetric instances based on network eli76 and 10 asymmetric instances based on network ftv64, and begin by solving each instance with intermediate values of $Q=4$ and $B=4$. We then vary $B$ and $Q$ to determine the impact that these parameters have on computational time and objective function value. For this experiment we use the twophase LKH algorithm since it is the best performer among the proposed algorithms. Table 3 presents the results of this experiment.

Table 3: Measuring the effect of $Q$ and $B$ on execution time and objective

\begin{tabular}{rrrrrlrrrr}
\hline$Q$ & $B$ & \multicolumn{4}{c}{ eli76 (symmetric) } & \multicolumn{3}{c}{ ftv64 (asymmetric) } \\
\cline { 2 - 9 } & & Avg & Max & \# solved & $z^{*}$ & Avg & Max & \# solved & $z^{*}$ \\
\hline 0 & 4 & 20 & 57 & 10 & 560 & 21 & 43 & 10 & 1933 \\
2 & 4 & 63 & 134 & 10 & 558 & 95 & 238 & 10 & 1915 \\
4 & 4 & 133 & 382 & 10 & 556 & 242 & 467 & 10 & 1907 \\
6 & 4 & 276 & 699 & 10 & 554 & 454 & 723 & 10 & 1899 \\
8 & 4 & 352 & 486 & 10 & 553 & 676 & 1087 & 10 & 1892 \\
10 & 4 & 679 & 1127 & 10 & 552 & 1004 & 1501 & 10 & 1887 \\
& & & & & & & & & \\
4 & 2 & 14 & 18 & 10 & 547 & 67 & 130 & 10 & 1875 \\
4 & 4 & 132 & 381 & 10 & 556 & 241 & 464 & 10 & 1907 \\
4 & 6 & 2193 & 5874 & 10 & 563 & 594 & 832 & 10 & 1935 \\
4 & 8 & 5823 & $>14,400$ & 6 & $570^{\dagger}$ & 1641 & 3354 & 10 & 1959 \\
\hline
\end{tabular}

$\dagger$ : Average optimal objective value computed only among the instances solved within the time limit. 
Table 3 shows that increasing the attacker's budget has a dramatic effect on the execution time of the algorithm. For the symmetric instances, the computational times increase from 14 seconds to over 2000 seconds as $B$ grows from 2 to 6 , and only six out of ten instances are solved to optimality when $B=8$. For the asymmetric instances the computational time increases by roughly a factor of three when increasing $B$ by two units. On the other hand, increasing the defender's budget has a less pronounced effect on the computational time. Increasing the defender's budget by ten units decreases $z^{*}$ by about $1.5 \%$ for the symmetric instances and $2.4 \%$ for the asymmetric instances. Increasing the attacker's budget by six units results in an objective value increase of roughly $4 \%$ for both the symmetric and the asymmetric instances.

We also conduct experiments to measure the effect of increasing the arc delay coefficient range on the execution times. For this purpose, we generate new instances based on symmetric network bayg29 and asymmetric network p43. We generate 20 instances (10 symmetric and 10 asymmetric) having random arc delay coefficients uniformly distributed between $\left[1,2 c_{i j}\right], 20$ instances having delay coefficients between $\left[1,3 c_{i j}\right]$, and 20 instances having delay coefficients between $[1, M]$, where $M=10 \max _{(i, j) \in \mathcal{A}}\left\{c_{i j}\right\}$. The latter delay configuration models the case in which an arc may become unavailable when interdicted. Table 4 presents results over this new set of instances. As before, we use intermediate values of $Q=4$ and $B=4$.

Table 4: Measuring the effect of varying the delay coefficient range on execution time

\begin{tabular}{rrrrrrrrr}
\hline Delay configuration & \multicolumn{3}{c}{ bayg29 (symmetric) } & \multicolumn{4}{c}{ p43 (asymmetric) } \\
\cline { 2 - 9 } & Avg & Max & \# solved & $z^{*}$ & Avg & Max & \# solved & $z^{*}$ \\
\hline$\left[1, c_{i j}\right]$ & 25 & 46 & 10 & 1686 & 185 & 363 & 10 & 5624 \\
{$\left[1,2 c_{i j}\right]$} & 119 & 173 & 10 & 1712 & 214 & 357 & 10 & 5624 \\
{$\left[1,3 c_{i j}\right]$} & 344 & 875 & 10 & 1722 & 225 & 337 & 10 & 5626 \\
{$[1, M]$} & $>14,400$ & $>14,400$ & 0 & - & 749 & 1505 & 10 & 5633 \\
\hline
\end{tabular}

Table 4 shows that for the symmetric instances, the computational time increases by roughly a factor of 15 as the delay range increases from $\left[1, c_{i j}\right]$ to $\left[1,3 c_{i j}\right]$, and none of the instances for which the delay coefficients are between $[1, M]$ terminate within the four-hour time limit. On the contrary, for the asymmetric instances the computational time exhibits a moderate increase with respect to the delay coefficient range and the instances having delay 
coefficients between $[1, M]$ are solved on average in about 12 minutes. The increased difficulty of solving symmetric instances as the range of the $d$-parameters grows may be due to the amount of similar tours that exist in the sample, which we expect to be considerably greater for symmetric instances than for asymmetric instances.

To mitigate the extent to which similar tours are included in the sample, we modify the objective function in phase one of our algorithm to include a penalty function based on sample diversity. Let $u_{i j}$ be the number of tours in the sample that use arc $(i, j)$. For a given attack $\hat{\mathbf{x}}$, we set the third-stage objective function in phase one as

$$
\min _{\mathbf{y} \in \mathcal{Y}} \sum_{(i, j) \in \mathcal{A}}\left(c_{i j}+d_{i j} \hat{x}_{i j}\right) y_{i j}+P u_{i j} y_{i j}
$$

where $P$ is an arbitrary constant penalty. After fine-tuning the two-phase LKH algorithm, we set $P$ equal to $1 / 2$.

Table 5: Imposing a penalty on the number of times an arc is used in the sample

\begin{tabular}{rrrrrrrrr}
\hline Delay configuration & \multicolumn{3}{c}{ bayg29 (symmetric) } & \multicolumn{5}{c}{ p43 (asymmetric) } \\
\cline { 2 - 9 } & Avg & Max & \# solved & $z^{*}$ & Avg & Max & \# solved & $z^{*}$ \\
\hline$\left[1, c_{i j}\right]$ & 39 & 65 & 10 & 1686 & 346 & 607 & 10 & 5624 \\
{$\left[1,2 c_{i j}\right]$} & 122 & 184 & 10 & 1712 & 455 & 645 & 10 & 5624 \\
{$\left[1,3 c_{i j}\right]$} & 250 & 520 & 10 & 1722 & 462 & 553 & 10 & 5626 \\
{$[1, M]$} & 5180 & 6826 & 10 & 1759 & 1562 & 1886 & 10 & 5633 \\
\hline
\end{tabular}

Table 5 shows that including the penalty in the objective function greatly reduces the computational time for solving the symmetric instances having delay coefficients between $[1, M]$, which are now solved in less than 2 hours. On the other hand, including the penalty in the objective function has a negative effect on the execution time for the asymmetric instances, which in the worst case increases by roughly a factor of 2 .

\section{Acknowledgments}

The authors are grateful for the remarks of an anonymous referee and an Associate Editor, whose comments led to an improved version of this paper. The authors also gratefully acknowledge the support of the Air Force Office of Scientific Research under grant FA9550- 
12-1-0353, and the Office of Naval Research under grant N000141310036.

\section{References}

[1] M. M. Flood, The traveling-salesman problem, Operations Research 4 (1) (1956) 61-75.

[2] E. L. Lawler, J. K. Lenstra, A. H. G. Rinnooy Kan, D. B. Shmoys, The Traveling Salesman Problem: A Guided Tour of Combinatorial Optimization, John Wiley \& Sons, New York, 1985.

[3] V. Pillac, M. Gendreau, C. Guéret, A. L. Medaglia, A review of dynamic vehicle routing problems, European Journal of Operational Research 225 (1) (2013) 1-11.

[4] P. Cappanera, M. P. Scaparra, Optimal allocation of protective resources in shortestpath networks, Transportation Science 45 (1) (2011) 64-80.

[5] R. L. Church, M. P. Scaparra, The $r$-interdiction median problem with fortification, Geographical Analysis 39 (2) (2007) 129-146.

[6] M. P. Scaparra, R. L. Church, A bilevel mixed-integer program for critical infrastructure protection planning, Computers and Operations Research 35 (6) (2008) 1905-1923.

[7] M. P. Scaparra, R. L. Church, An exact solution approach for the interdiction median problem with fortification, European Journal of Operational Research 189 (1) (2008) 76-92.

[8] J. R. O'Hanley, R. L. Church, Designing robust coverage networks to hedge against worst-case facility losses, European Journal of Operational Research 209 (1) (2011) 2336 .

[9] S. Starita, M. P. Scaparra, Optimizing dynamic investment decisions for railway systems protection, European Journal of Operational Research 248 (2) (2016) 543-557.

[10] G. G. Brown, W. M. Carlyle, J. Salmerón, R. K. Wood, Defending critical infrastructure, Interfaces 36 (6) (2006) 530-544.

[11] M. Prince, J. C. Smith, J. Geunes, A three-stage procurement optimization problem under uncertainty, Naval Research Logistics 60 (1) (2013) 395-412.

[12] J. C. Smith, C. Lim, F. Sudargho, Survivable network design under optimal and heuristic interdiction scenarios, Journal of Global Optimization 38 (2) (2007) 181-199.

[13] L. Lozano, J. C. Smith, A backward sampling framework for interdiction problems with fortification, Technical report, Department of Industrial Engineering, Clemson University, Clemson, SC (2016).

[14] E. Balas, R. Jeroslow, Canonical cuts on the unit hypercube, SIAM Journal on Applied Mathematics 23 (1) (1972) 61-69.

[15] K. Deb, A. Pratap, S. Agarwal, T. Meyarivan, A fast and elitist multiobjective genetic algorithm: NSGA-II, IEEE Transactions on Evolutionary Computation 6 (2) (2002) $182-197$.

[16] R. K. Ahuja, Ö. Ergun, J. B. Orlin, A. P. Punnen, A survey of very large-scale neighborhood search techniques, Discrete Applied Mathematics 123 (1) (2002) 75-102.

[17] S. Lin, B. W. Kernighan, An effective heuristic algorithm for the traveling-salesman problem, Operations Research 21 (2) (1973) 498-516. 
[18] E. Israeli, R. K. Wood, Shortest-path network interdiction, Networks 40 (2) (2002) 97111.

[19] D. Applegate, R. Bixby, V. Chvátal, W. Cook, CONCORDE TSP solver, available at www. math. uwaterloo.ca/tsp/concorde.html.

[20] D. Applegate, R. Bixby, W. Cook, V. Chvátal, On the solution of traveling salesman problems, Rheinische Friedrich-Wilhelms-Universität, Bonn, Germany, 1998.

[21] K. Helsgaun, An effective implementation of the Lin-Kernighan traveling salesman heuristic, European Journal of Operational Research 126 (1) (2000) 106-130.

[22] G. Reinelt, TSPLIB-A traveling salesman problem library, INFORMS Journal on Computing 3 (4) (1991) 376-384. 